\title{
Wigner energy, odd-even mass staggering and the time-odd mean-fields ${ }^{1}$
}

\author{
Wojciech Satuła \\ Institute of Theoretical Physics, University of Warsaw, ul. Hoża 69, 00-681 Warszawa, Poland \\ Joint Institute for Heavy Ion Research, Oak Ridge, TN 37831-6374, USA \\ Department of Physics, University of Tennessee, Knoxville, TN 37996, USA
}

\begin{abstract}
Various properties of single-particle Hartree-Fock ground-state solutions in $N \sim Z$ nuclei are investigated. The emphasis is on a role of single-particle mean-field in odd-even mass staggering. It is shown that, unlike in traditional scenario originating from the Fermi gas or macroscopic models, the symmetry energy contribution to odd-even mass staggering is nearly cancelled by the contribution coming from the average level density. It allows to construct indicators probing both pairing as well as mean-field components to the oddeven mass staggering. The impact of the single-particle Hartree-Fock field on Wigner energy and residual pn interaction in odd-odd nuclei is also discussed.
\end{abstract}

\section{INTRODUCTION}

Mean-field is considered as a standard nuclear model to describe medium mass and heavy nuclei. Indeed, various applications demonstrated ability of this relatively simple concept to describe wide range of nuclear phenomena. However, the accuracy of mean-field results or predictions is far from satisfactory when confronted with the present day, high precision experimental data. The predictions in many cases differ substantially for various parametrizations of the same effective interaction and the situation calls for systematic program to optimize the forces. Recent work of Chabanat et al. [1] can be considered as the first step towards finding comprehensive parametrization of the Skyrme force [2]. Indeed, the so called Lyon (SLy) forces developed in Ref. [1] are very widely used and are considered to be one of the best among the Skyrme forces available nowadays.

The aim of this work is to search for certain generic features of the ground-state Skyrme-Hartree-Fock (SHF) solutions which may help in better understanding of basic properties of this force through the nature of self-consistent solutions. First section discusses a role of single-particle SHF mean-field in odd-even mass staggering (OES) which, in atomic nuclei, is usually attributed to pairing [3,4]. Proper understanding of mean-field and pairing contributions to OES is crucial to better understand and parametrize both channels. The second section investigates the possibility to obtain an additional binding energy in the $N=Z$ nuclei within single-particle self-consistent SHF model or conventional, i.e. including only $T=1,\left|T_{z}\right|=1$ pairing correlations, self-consistent Hartree-Fock-Bogolyubov model. Finally, the third section discusses properties of self-consistent SHF solutions in time-odd channel.

\section{ODD-EVEN MASS STAGGERING}

The odd-even mass staggering in atomic nuclei is usually attributed to the presence of pairing correlations [3]. Indeed, in the simplest scenario based on standard BCS theory of superfluidity [5], the ground state energies of three adjacent isotopes (isotones) can be approximately interrelated by a simple readjustment of their chemical potentials $\lambda$ :

$$
B(N), \quad B(N \pm 1) \approx B(N)+E_{k} \pm \lambda,
$$

where $E_{k} \approx \Delta$ denotes energy of the lowest quasiparticle in odd- $N$ nucleus. Consequently, the quantity

$$
\Delta_{\nu}^{(3)}(N)=\frac{\pi_{N}}{2}(B(N-1)-2 B(N)+B(N+1)) \approx E_{k} \approx \Delta,
$$

1) Plenary talk presented at Nuclear Structure'98, Gatlinburg, Tenn., USA, August 10-15, 1998 


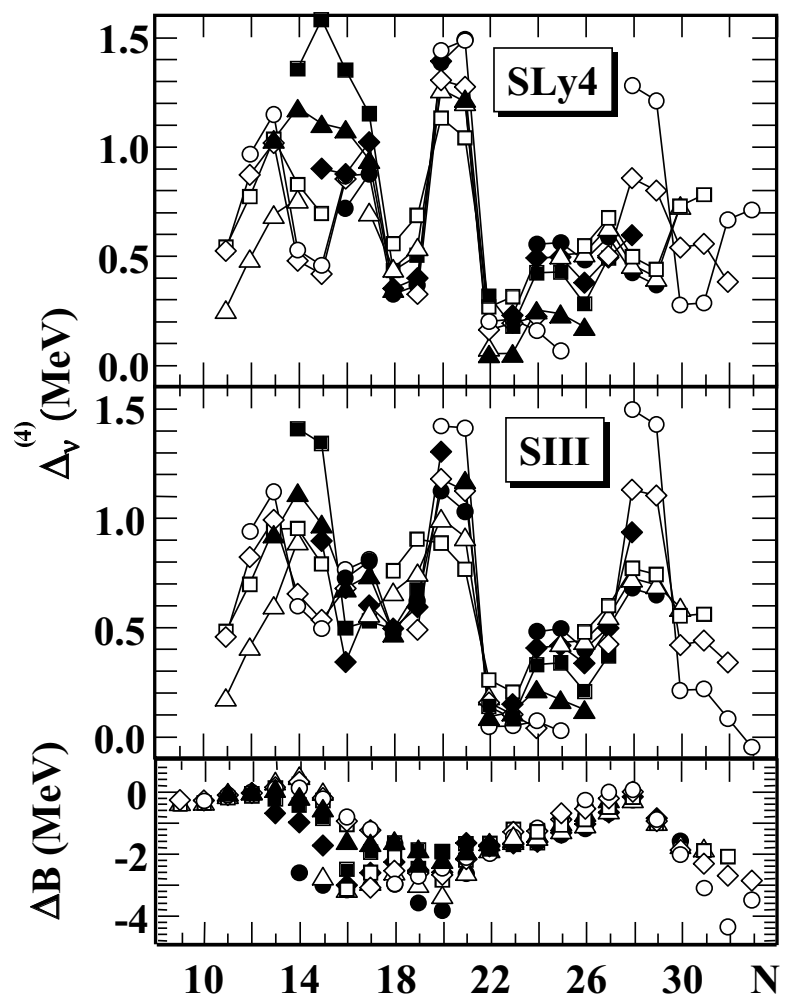

FIGURE 1. The OES $\Delta_{\nu}^{(4)}$ as a function of neutron number $N$ computed from theoretical masses calculated using SHF theory with SLy4 (upper part) and SIII (middle part) Skyrme interaction. Note that the pattern of OES is almost independent on the interaction. The lowest panel shows the difference of nuclear masses calculated in both cases. Unlike the OES (local mass correlations) calculated masses are strongly interaction dependent.

is often interpreted as a measure of empirical pairing gap, $\Delta[6]$. In Eq. $(2), \pi_{N}=(-1)^{N}$ is the number parity and $B(N)$ is the (negative) binding energy of the system with $N$ particles. It has been noticed long time ago based on the Fermi gas model that the quantity (2) contains strong contribution from the symmetry energy [7]. Because $\Delta_{\nu}^{(3)}(N)$ is a finite step approximation of derivative of the second order:

$$
\Delta_{\nu}^{(3)}(N) \approx \frac{\partial^{2} B(N)}{\partial N^{2}}
$$

the symmetry energy $\left[\sim a_{I}(A)(N-Z)^{2}\right]$ will indeed strongly contribute to OES according to this criterion. However, this contribution is independent on nucleon-number parity and, therefore, the symmetry energy effect can be removed simply by replacing the three-point indicator (2) by the higher order expression like the one containing four masses [8]:

$$
\begin{aligned}
\Delta^{(4)}(N) & \equiv \frac{\pi_{N}}{4}[3 B(N-1)-3 B(N)-B(N-2) \\
& +B(N+1)]=\frac{1}{2}\left[\Delta^{(3)}(N)+\Delta^{(3)}(N-1)\right] .
\end{aligned}
$$

Consequently, in the traditional picture originating from the Fermi gas or liquid drop (macroscopic) models the meanfield contribution to the odd-even mass staggering is predominantly due to symmetry energy and can essentially be removed by means of high-order indicators like (4), see [9] and refs. quoted therein.

Contrary to the above so far commonly accepted scenario, the indicator (4) generates sizable OES when applied to the mass table calculated using single-particle Skyrme-Hartree-Fock model, see [4] and Fig. 1. These mass 


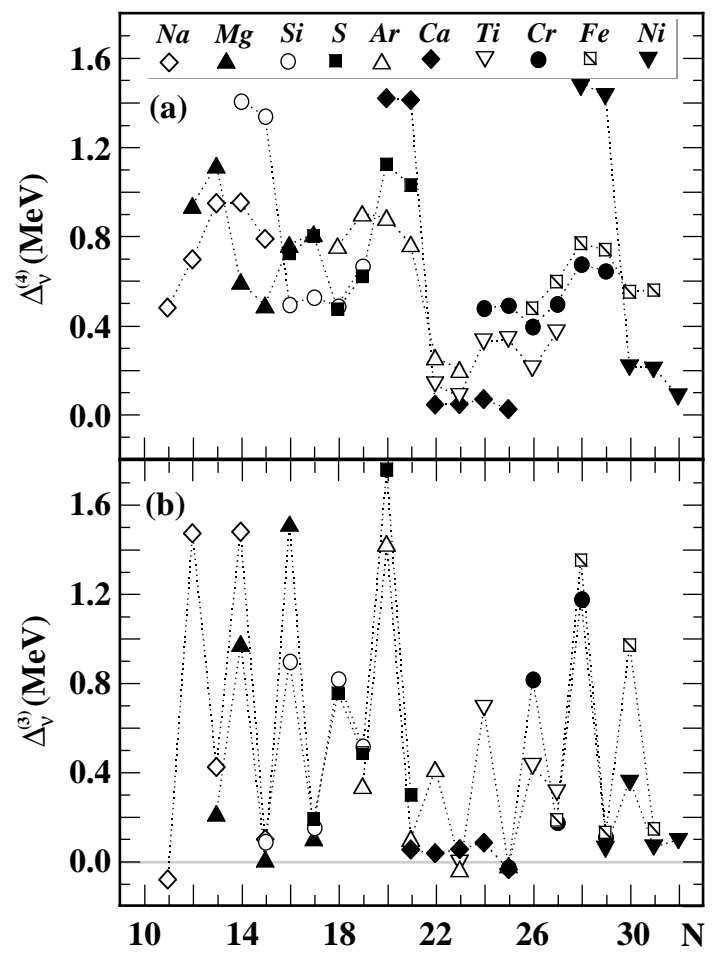

FIGURE 2. The OES $\Delta_{\nu}^{(4)}$ (upper part) and $\Delta_{\nu}^{(3)}$ (lower part) computed from theoretical masses calculated using SHF theory with SIII Skyrme interaction. Note, that rather complicated pattern of OES $\Delta_{\nu}^{(4)} \operatorname{simplifies}$ for $\Delta_{\nu}^{(3)}$. The $\Delta_{\nu}^{(3)}$ shows clear alternating pattern with large values for even- $N$ and small for odd- $N$. Taken from [4].

calculations has been performed for nuclei with $9 \leq Z \leq 28$ and $-2 \leq N-Z \leq 6$, using HFODD code (v1.75) of [10] and two parametrizations of the Skyrme force: SIII [11] and SLy4 [1]. The pattern of OES, which reaches from 30\% to $50 \%$ of experimental value, appears to be generic feature of the SHF solutions i.e. is almost independent on parametrization although calculated masses differ substantially for the two parametrizations employed, see Fig. 1.

A rather complicated pattern of OES in Fig. 1 simplifies when criterion $\Delta^{(3)}$ is used instead of $\Delta^{(4)}$, see Fig. 2. Indeed, values of $\Delta^{(3)}(N)$ are large for even- $N$ and small (close to zero) for odd- $N$. Furthermore, it appears possible to explain this alternating $\Delta^{(3)}(N)$ behavior obtained directly in fully microscopic SHF calculations by using simple arguments based on Strutinsky energy theorem [12]. According to this theorem the results of selfconsistent calculations can be well approximated by the microscopic-macroscopic shell-correction method i.e. the total binding energy can be written as $B=E_{\mathrm{sp}}-\tilde{E}_{\mathrm{sp}}+E_{\text {macro }}$, where

$$
E_{\mathrm{sp}}=\sum_{k=1}^{A} e_{k}
$$

is the shell-model energy (sum of single-particle energies of occupied states), $\tilde{E}_{\mathrm{sp}}$ is the Strutinsky-averaged shellmodel energy, and $E_{\text {macro }}$ stands for the macroscopic liquid-drop energy.

The contribution to OES from shell-model energy is:

$$
\delta e \equiv \Delta_{\mathrm{sp}}^{(3)}(N) \approx \frac{1}{4}\left(1+\pi_{N}\right)\left(e_{n+1}-e_{n}\right)=\left\{\begin{array}{rll}
0 & \text { if } & N=2 n+1 \\
\left(e_{n+1}-e_{n}\right) & \text { if } & N=2 n
\end{array}\right.
$$

This single-particle mechanism behind OES was first noticed in Ref. [13] and applied to OES in metal clusters [14-16]. The contribution coming from smooth Strutinsky energy can be expressed through average level density at the Fermi energy $g(\lambda)$ :

$$
\Delta^{(3)}(N) \approx \frac{\pi_{N}}{2 g(\lambda)} .
$$

The average single-particle level density is $g(\lambda)=3 a / \pi^{2}\left(m^{*} / m\right)$ [13]. The empirical value of the level density parameter for light nuclei is $a \approx A / 8 \mathrm{MeV}$ what agrees well with the estimate based on realistic potentials [17]. 


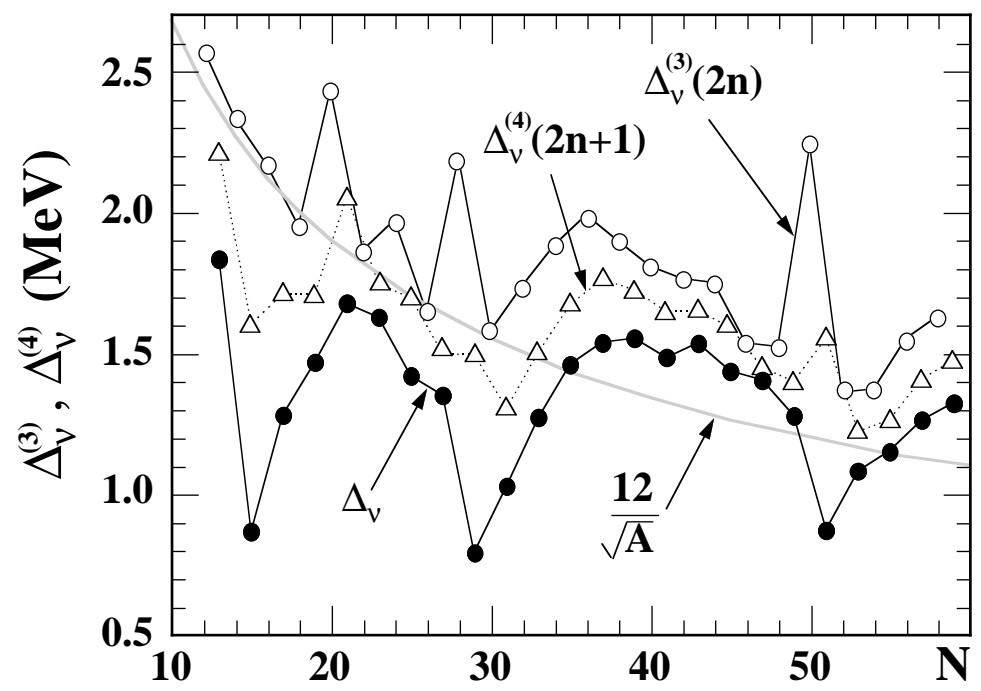

FIGURE 3. The empirical values of $\Delta_{\nu}^{(3)}(N=2 n+1)\left[\equiv \Delta_{\nu}\right], \Delta_{\nu}^{(3)}(N=2 n)$, and $\Delta_{\nu}^{(4)}(N=2 n+1)$ as a function of neutron number. Each point represents average mean over several even- $Z$ isotones. Note, that only $\Delta_{\nu}^{(3)}(N=2 n+1)$ shows characteristic quenching at (semi)magic gaps at $N=14,28,50$ (no effect at $N=20$ is seen) as anticipated for pairing. Thick gray line shows the average trend $\Delta=12 / \sqrt{A} \mathrm{MeV}$. Taken from Ref. [4].

A correction due to effective mass is $m^{*} / m=0.76(0.70)$ for SIII and SLy4 forces, respectively. Consequently, the contribution to OES coming from smooth Strutinsky energy equals $\delta \Delta^{(3)} \approx-18 / A \mathrm{MeV}$. Contribution from macroscopic energy is essentially due to symmetry energy $\left[\frac{a_{I}}{2 A}(N-Z)^{2}\right]$. The empirical value of the symmetry energy strength in light nuclei is $a_{I}=38 \mathrm{MeV}$ [23]. Hence, the liquid-drop contribution to OES is $\delta \Delta^{(3)} \approx 19 / A \mathrm{MeV}$ what indeed nearly cancels out the contribution coming from the smooth Strutinsky energy.

The alternating pattern of $\Delta^{(3)}(N)$ allows to extract both pairing and mean-field component of OES. Indeed, at odd- $N$ mean-field component to $\Delta^{(3)}(N)$ is small and its value can be associated mainly with pairing:

$$
\Delta_{\nu}(N) \equiv \Delta_{\nu}^{(3)}(N=2 n+1),
$$

while the differences:

$$
e_{n+1}-e_{n}=2\left[\Delta_{\nu}^{(3)}(N=2 n)-\Delta_{\nu}^{(3)}(N=2 n+1)\right],
$$

provide information about effective Nilsson single-particle spectra [for more details concerning this aspect see discussion and Fig. 4 in Ref. [4]].

Empirical values of $\Delta_{\nu}^{(3)}$ and $\Delta_{\nu}^{(4)}$ are shown in Fig. 3. Note, that only values of $\Delta_{\nu}^{(3)}(N=2 n+1)$ show oscillatory behavior reflecting expected quenching of neutron pairing correlations at the magic (semi-magic) gaps at $N=14,28$, and 50. Rather surprisingly no quenching effect is seen at $N=20$. For $\Delta_{\nu}^{(3)}(2 n)$ and $\Delta_{\nu}^{(4)}$ the expected quenching of pairing correlations is counterbalanced by single-particle effects and no minima are seen in these cases. These empirical observations neatly support the above scenario.

Similar interpretation of quantities (8) and (9) can be drown also based on seniority, equidistant-level [Richardson], and pairing-plus-quadrupole models [18]. Let's consider here as an example a seniority (or pairing quasispin) model. The ground state energy in this model [see e.g. [6] p. 222] equals:

$$
B(N, s)=-\frac{G}{4}(N-s)(2 \Omega-s-N+2) \text { where }\left\{\begin{array}{lll}
s=0 & \text { for } \quad N=2 n \\
s=1 & \text { for } \quad N=2 n+1
\end{array},\right.
$$

where $s$ is a seniority quantum number, $\Omega$ stands for degeneracy of the shell and $G$ denotes pairing strength. After simple algebra one gets:

$$
\Delta^{(3)}(N)=\left\{\begin{array}{lll}
\frac{1}{2} G \Omega+\frac{1}{2} G & \text { for } & N=2 n \\
\frac{1}{2} G \Omega & \text { for } & N=2 n+1
\end{array} .\right.
$$




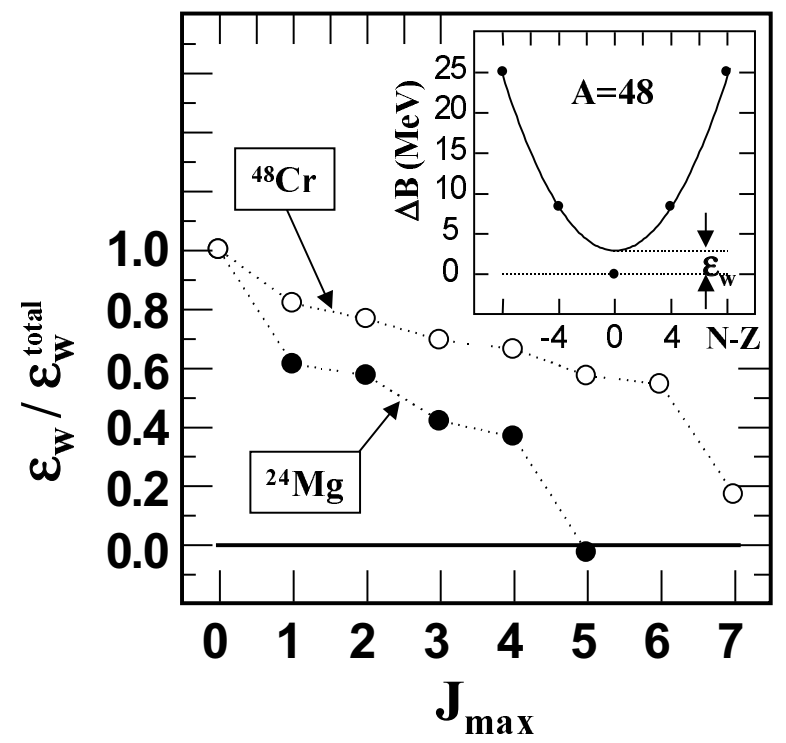

FIGURE 4. The contributions to the energy $\varepsilon_{W}$ coming from isoscalar pairs of different angular momenta in ${ }^{24} \mathrm{Mg}$ (solid circels) and ${ }^{48} \mathrm{Cr}$ (open circels). The energy $\varepsilon_{W}$ defines additional binding energy in $N=Z$ nucleus as a displacement of its binding energy from the average parabolic $\sim(N-Z)^{2}$ behavior in the $N \neq Z$ isostones, see inset. The details of this $0 \hbar \omega$ shell-model calculation can be found in Ref. [22].

Note that in accordance with (8) indicator $\Delta^{(3)}(N=2 n+1)$ probes only collective pairing energy while $\Delta^{(3)}(N=2 n)$ contains also weak, $\delta e=G$ according to (9), mean-field contribution.

In the BCS approximation single-particle potential contributes both to $\Delta^{(3)}(N=2 n)$ and $\Delta^{(3)}(N=2 n+1)$. In this case one obtains Eq. (11) only in open shell regime where number of pairs is $n \sim \Omega / 2 \gg 1$. In this regime, where BCS is considered to be very good approximation, also $\Delta_{B C S} \approx G \Omega / 2\left[\equiv \Delta^{(3)}(2 n+1)_{\text {exact }}\right]$.

\section{WIGNER ENERGY}

It has been known since the early work of [19] that macroscopic-microscopic approaches systematically underbinds $N \approx Z$ nuclei. Similar situation holds in semi-classical Thomas-Fermi models [20,21] as well as in spherical SkyrmeHartree-Fock calculations irrespectively on parametrization of the Skyrme force $[22,23]$. This extra binding energy, which is characterized by $\sim|N-Z|$ type singularity at the $N=Z$ line, is dubbed Wigner energy and is usually parametrized as:

$$
E_{W}=W(A)|N-Z|+d(A) \pi_{p n} \delta_{N Z},
$$

where $W(A)$ and $(A)$ are smooth functions of mass, and $\pi_{p n}=1(0)$ for odd-odd(other) nuclei. Parametrization (12) can be justified based on simple arguments of counting of $n p$-pairs in identical spatial orbitals and on elementary properties of $n p$ interaction [24,9]. This estimate gives also $W / d=1$. In Ref. [22] (for more details see [23]) we constructed a family of indicators able to probe both $W(A)$ and $d(A)$. It appears that indeed $W(A) \approx d(A)$ provided that the energies of the lowest $T=0$ states are used in these indicators instead of masses of heavy $N=Z$ odd-odd nuclei. It provides independent empirical argument that Wigner energy is indeed predominantly related to $T=0 n p$-interaction.

Unlike mean-field, the state-of-the-art shell-model calculations reproduce empirical Wigner energy very well [22]. One can, therefore, use this model to gain some knowledge about microscopic structure of the Wigner energy. The shell-model indeed relates the Wigner energy to $T=0$ interaction. It has been demonstrated first by Brenner et 


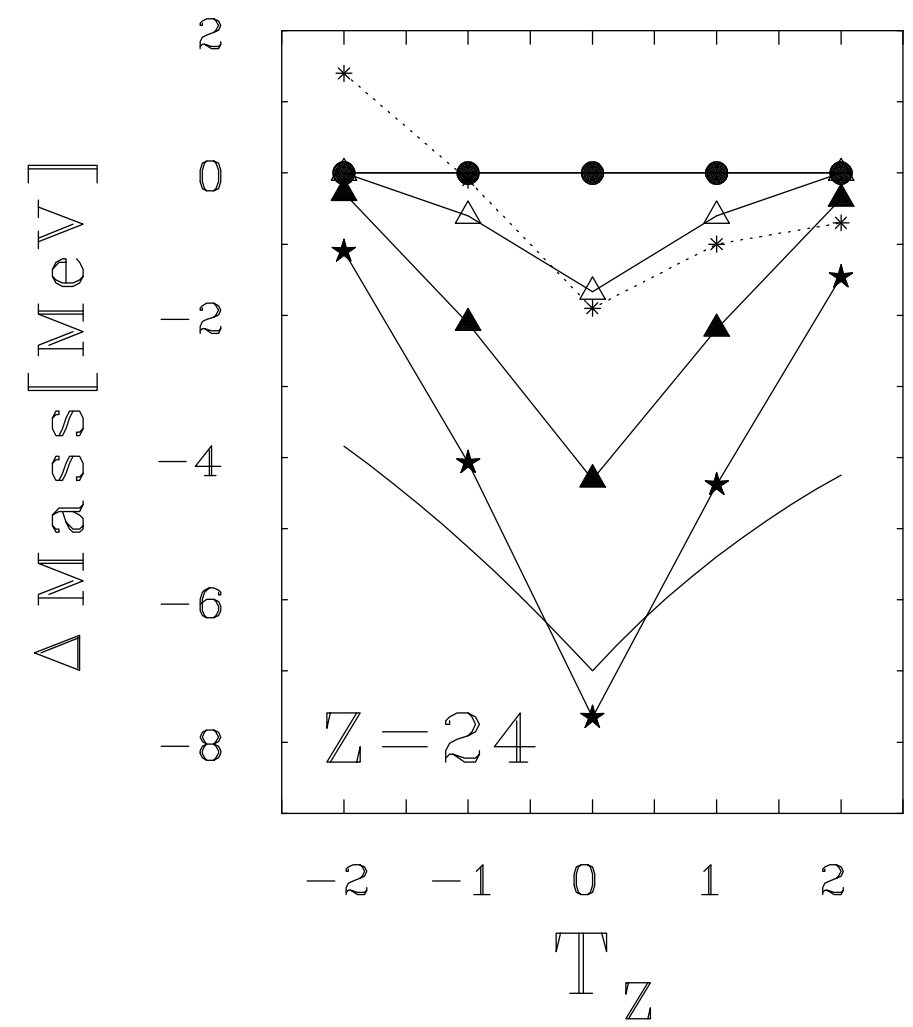

FIGURE 5. The mass excess $\Delta M=B\left(x^{T}\right)-B\left(x^{T}=1\right)$ calculated using generalized BCS plus Lipkin-Nogami theory as a function of $T_{z}$. Different curves correspond to different values of $x^{T}$ being a ratio of the isoscalar to the isovector coupling constants. Dotted line marks the results of extended Thomas-Fermi calculations [20] while solid line without symbols Wigner energy according to formula from Ref. [19]. Figure taken from Ref. [26].

al. [25] in $s d$ shell. The detailed microscopic analysis in $s d$ and $f p$ shells [22] revealed, however, rather complex structure of the Wigner energy in terms of nucleonic isoscalar pairs of different angular momenta. Although the largest contributions come from pairs coupled to $J=1$ and $J=J_{\max }=5(7)$ for $s d$ and $f p$ shells, respectively, all matrix elements of intermediate angular momenta seem to be rather important, see Fig. 4.

The deficiencies of conventional mean-field which allows only for $T=1,\left|T_{z}\right|=1$ pairing can be remedied in generalized mean-field theory which takes into account also $n p$-pairing correlations. It has been demonstrated in Ref. [26] that isoscalar $n p$-pairing correlations can provide missing binding energy at $N=Z$ line and its closest vicinity as shown in Fig. 5. The model Hamiltonian employed in [26] was very simple, based on similar pair counting mechanism like discussed above in connection with the Wigner energy parametrization. For such a simple Hamiltonian generalized BCS solutions do not mix $T=0$ and $T=1$ pair correlations (see also [32]) and the energy gain is possible only when the isoscalar correlations are on the average stronger than the isovector. The latter cannot give an extra binding energy (in even-even nuclei) as long as isospin is (approximately) conserved. Simple estimates of isoscalar and isovector pair correlations in simple nuclei like ${ }^{42} \mathrm{Sc}$ suggest indeed that isoscalar correlations are on the average stronger than isovector, see discussion in $[27,28]$. However, connection between empirical interactions deduced from the spectra of simple nuclei and the mean-field effective pairing interaction is not obvious. Fast disappearance of isoparing with increasing $T_{z}=(N-Z) / 2$ shows up naturally in generalized BCS model and can be easily understood in terms of blocking of $n p$-pairing due to proton or neutron excess [26,29]. One should mention here, that recent generalized BCS calculations with $G$-matrix interaction [31] in $f p g$ shell $(A \sim 80)$ do allow for mixing of $T=0$ and $T=1$ pair correlations similar to the shell-model solutions [32].

The $n p$-pairing is not necessarily the only contribution to the Wigner energy. In principle also self-consistent meanfield itself can contribute to the extra binding in $N=Z$ nuclei because of congruent nodal structure of a neutron and proton wave functions which can lead to stronger $n p$-interaction as suggested by [21]. The congruence energy can manifest itself only in fully microscopic models. Spherical, conventional Hartree-Fock-Bogolyubov calculations with $T=1,\left|T_{z}\right|=1$ pairing only do not show any congruence effects as shown in Fig. 6, see also Refs. [22,23]. This result neither depends on Skyrme interaction nor pairing force. However, deformed SHF-plus-BCS calculations do show around $\sim 30 \%$ of the empirical Wigner energy. Both spherical and deformed calculations are illustrated in 


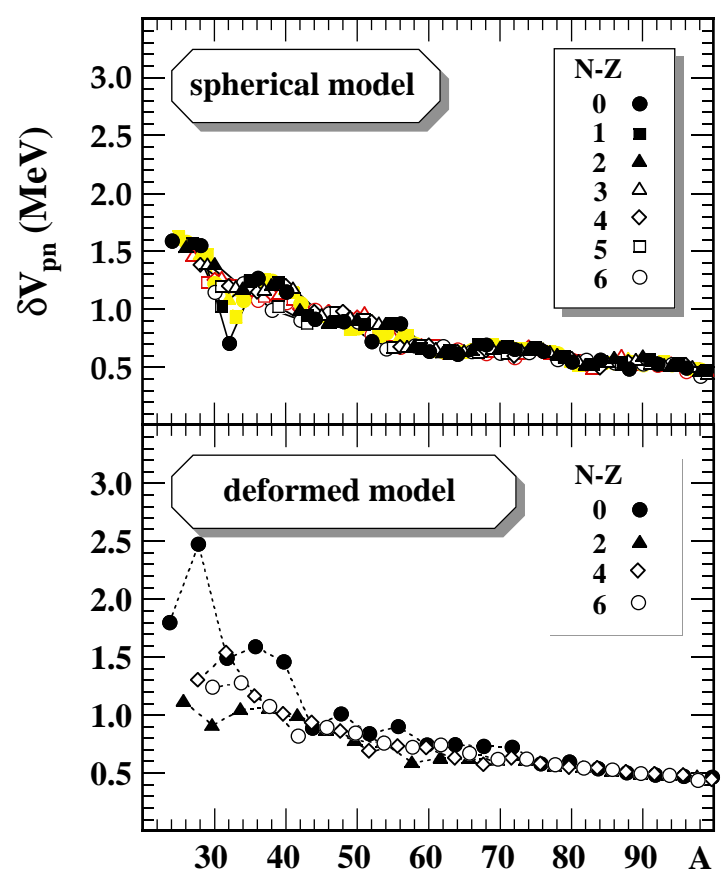

FIGURE 6. The values of $\delta V_{p n}$ (see Eq. (13)) calculated using theoretical masses computed by means of spherical SHF model with SLy4 Skyrme force and density dependent, surface active delta interaction in pairing channel (upper part) and theoretical masses computed by Tajima et al. [30] using deformed SHF-plus-BCS theory with SIII Skyrme interaction (lower part). Figure taken from [23].

Fig. 6 which shows values of the so called double-difference indicator [35]

$$
\delta V_{n p}=[B(N, Z)-B(N-2, Z)-B(N, Z-2)+B(N-2, Z-2)] / 4 \approx \frac{\partial^{2} B(N, Z)}{\partial N \partial Z} .
$$

This quantity is particularly convenient to probe Wigner energy [22]. In deformed calculations (only even-even nuclei) masses computed by Tajima et al. [30] were used to calculate $\delta V_{n p}$. Very similar effect (about $30 \%$ of experimental value) was obtained also in our single-particle deformed SHF calculations [23]. In these calculations also odd-odd nuclei has been included. Rather surprisingly, an enhancement of $\delta V_{n p}$ in odd-odd $N=Z$ nuclei comes entirely from the time-odd isoscalar field, see next section for greater detail.

\section{THE TIME-ODD MEAN-FIELDS}

Investigation of odd and odd-odd nuclei require the time-odd mean-fields to be systematically taken into account in the calculations. The time-odd part of the energy density $\mathcal{H}^{\text {odd }}$ reads:

$$
\mathcal{H}_{t}^{o d d}=C_{t}^{s} \boldsymbol{s}_{t}^{2}+C_{t}^{\Delta s} \boldsymbol{s}_{t} \cdot \boldsymbol{\Delta} \boldsymbol{s}_{\boldsymbol{t}}+C_{t}^{T} \boldsymbol{s}_{\boldsymbol{t}} \cdot \boldsymbol{T}_{\boldsymbol{t}}+C_{t}^{j} \boldsymbol{j}_{t}^{2} C_{t}^{\nabla j} \boldsymbol{s}_{\boldsymbol{t}} \cdot\left(\nabla \times \boldsymbol{j}_{\boldsymbol{t}}\right)
$$

where $\boldsymbol{s}, \boldsymbol{j}, \boldsymbol{T}$ are time-odd spin, momentum, and vector kinetic densities, respectively, and subscript $t[=0,1]$ denotes isospin $[33,10]$.

The effective contribution of the time-odd mean-fields to the total energy $E^{t o}$ can be investigated by comparing full SHF calculations with the calculations restricted to time-even fields only. The result is shown in Fig. 7 . As seen from the figure everywhere beyond $N=Z$ line $E^{\text {to }}$ is small but force dependent. Indeed, the time-odd fields are effectively attractive for SLy4 but repulsive for SIII forces, respectively. Also beyond $N=Z$ line simple additivity for the averages [see inset in Fig. 7]:

$$
\bar{E}^{t o}(N-Z=2 n) \approx \bar{E}^{t o}(N-Z=2 n-1)+\bar{E}^{t o}(N-Z=2 n+1),
$$




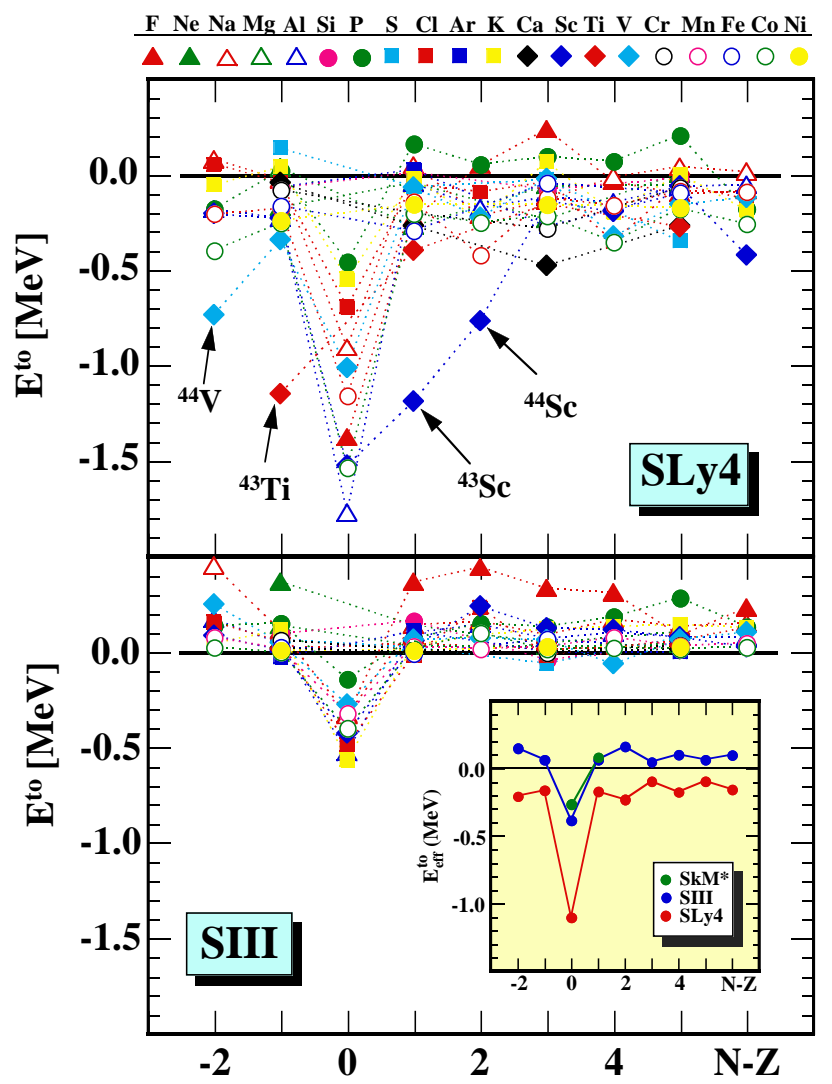

FIGURE 7. The effective energetical contribution to nuclear mass due to time-odd mean-field as a function of $N-Z$. The calculations were performed using SHF model with Sly4 (upper part) and SIII (lower part) Skyrme forces, respectively. An inset represents configuration independent contributions i.e. arithmetic averages over $T_{z}=$ const nuclei.

is rather well fulfilled, reflecting single-particle nature of the effect. The most remarkable observation is an enhancement of the time-odd effects in $N=Z$ nuclei. The effect is essentially due to cancellation of two large components in (14), namely a repulsive $C_{0}^{s} \boldsymbol{s}_{\mathbf{0}}^{\mathbf{2}}$ field is overbalanced by an attractive $C_{0}^{\Delta s} \boldsymbol{s}_{\mathbf{0}} \cdot \boldsymbol{\Delta} \boldsymbol{s}_{\mathbf{0}}$ field. The effect reflects most likely spontaneous spin polarization at the nuclear surface but its nature and consequences are not yet fully recognized and are under study [36]. It is noteworthy, that any observable consequences of this spin collectivity may help to establish certain constraints on time-odd coupling constants $C_{0}^{s}$ and $C_{0}^{\Delta s}$. Note that $C_{t}^{s}$ and $C_{t}^{\Delta s}$ are the only free time-odd coupling constants for Skyrme force. The remaining coupling constants (14) are related to time-even coupling constants because of local gauge invariance of the Skyrme force [34].

It is interesting to observe also strong instabilities in time-odd fields for $N \neq Z{ }^{43} \mathrm{Sc},{ }^{44} \mathrm{Sc}$ nuclei and in their isobaric analogs ${ }^{43} \mathrm{Ti},{ }^{44} \mathrm{~V}$. These instabilities show up only for SLy4 force and only at the very bottom of the $f_{7 / 2}$ shell. This is, most likely, due to the extended spatial dimensions of these nuclei and due to strong attractiveness of $C_{0}^{s}$ component of the SLy4 force at low densities where $C_{0}^{s}(\rho \rightarrow 0)=-207.8 \mathrm{MeV} \mathrm{fm}^{3}$. For most of the commonly used Skyrme forces $C_{0}^{s}(\rho \rightarrow 0)>0$, see table I in Ref. [33].

Let's finally consider briefly an indicator

$$
\begin{aligned}
\epsilon_{p n}=(-1)^{(A+1)}[-B(N-1, Z-1)+2 B(N, Z-1)-B(N+1, Z-1)+ \\
2 B(N-1, Z)-4 B(N, Z)+2 B(N+1, Z) \\
-B(N-1, Z+1)+2 B(N, Z+1)-B(N+1, Z+1)] / 4,
\end{aligned}
$$

which probes residual $p n$-interaction in odd-odd nuclei, see [9]. The data indicate, that for light $N \sim Z$ nuclei $\epsilon_{p n}$ is weekly dependent on nuclear mass and $\epsilon_{p n} \approx 500 \mathrm{keV}$. Only in the closest vicinity of $N=Z$ line $\epsilon_{p n}$ is enhanced due to the Wigner energy, see [22,23]. Applying the indicator (16) to single-particle SHF mass table gives no effect i.e. $\epsilon_{p n} \approx 0$ in odd-odd $N \neq Z$ nuclei. The cancellation of time-odd effects in (16) is a consequence of the above 
mentioned, see Eq. (15), energetical additivity of time-odd contributions in odd and odd-odd nuclei. Only in $N=Z$ odd-odd cases $\epsilon_{p n} \neq 0$ as a result of an enhancement of the time-odd fields in these nuclei. It seems therefore justified to conclude that the residual neutron-proton interaction in odd-odd nuclei as seen through the indicator (16) goes entirely beyond the mean-field.

\section{SUMMARY}

Selected properties of single-particle self-consistent Skyrme mean-field have been analyzed. It has been shown by direct microscopic calculations that the mean-field component to OES according to the criterion (2) shows strong nucleon number parity $\pi_{N(Z)}$ dependence with $\Delta_{\nu(\pi)}^{(3)}$ being large (small) for $\pi_{N(Z)}=+1(-1)$, respectively. This alternating pattern is due to $(i)$ cancellation of the contributions from symmetry energy and average level density, and $(i i)$ due to nuclear Jahn-Teller effect. It allows to interpret $\Delta_{\nu(\pi)}^{(3)}(N=2 n+1)$ directly as due to (almost) pure pairing and construct higher order indicator (9) to probe also the mean-field component to OES. This scenario is entirely different than the commonly accepted scenario derived from the Fermi gas model where mean-field component to OES is due to symmetry energy and thus is independent on the number parity.

It has been demonstrated that spherical HFB calculations including only $T=1,\left|T_{z}\right|=1$ pairing generate no congruence energy i.e. $W(A) \approx 0$. It appears also, that deformed single-particle SHF (or SHF-plus-BCS) mean-field can generate only relatively small fraction $\sim 30 \%$ of the empirical Wigner energy strength $W(A)$ in even-even $N=Z$ nuclei. Therefore, most of the Wigner energy strength in $N=Z$ nuclei seems to be beyond the Skyrme mean-field and, most likely, is due to neutron-proton pairing.

One of the most spectacular observations is strong enhancement of the time-odd effects in $N=Z$ nuclei. Closer examination shows that this effect depends essentially on the first two components (more precisely is due to their isoscalar parts) in (14). Because $C_{0}^{s}$ and $C_{0}^{\Delta s}$ are independent (free) time-odd coupling constants for Skyrme force any observable consequences of this effect can help to establish empirical constraints for their values. In contrast to the $N=Z$ nuclei, in $N \neq Z$ nuclei effective contributions to the total binding energy coming from the time-odd fields reflect simple additivity pattern between odd and odd-odd nuclei typical for a single-particle model. As a consequence, binding energy between valence proton and neutron in odd-odd nuclei meassured by means of the indicator (16) is zero. In other words $\epsilon_{p n}$ is entirely beyond SHF field.

\section{ACKNOWLEDGMENTS}

The material presented here was obtained in collaboration with D.J. Dean, J. Dobaczewski, W. Nazarewicz, and R. Wyss. This research was supported in part by the U.S. Department of Energy under Contract Nos. DE-FG0296ER40963 (University of Tennessee), DE-FG05-87ER40361 (Joint Institute for Heavy Ion Research), DE-AC0596OR22464 with Lockheed Martin Energy Research Corp. (Oak Ridge National Laboratory), and by the Polish Committee for Scientific Research (KBN) under Contract No. 2 P03B 04014.

\section{REFERENCES}

1. E. Chabanat E., Bonche P., Haensel P., Meyer J., and Schaeffer R., Nucl. Phys A627, 710 (1997).

2. Skyrme T.H.R., Phil. Mag. 1, 1043 (1956); Nucl. Phys. 9, 615 (1959).

3. Bohr A., Mottelson B.R., and Pines D., Phys. Rev. 110, 936 (1958).

4. Satuła W., Dobaczewski J., and Nazarewicz W., Phys. Rev. Lett. in print, and nucl-th/9804060

5. Bardeen J., Cooper L.N., and Schrieffer J.R., Phys. Rev. 110, 1175 (1957)

6. Ring P., and Schuck P., The Nuclear Many-Body Problem, Berlin: Springer-Verlag, (1980)

7. Bohr A., and Mottelson B.R., Nuclear Structure, vol. 2, New York: W.A. Benjamin, 1975.

8. Nilsson S.G., and O. Prior, Mat. Fys. Medd. Dan. Vid. Selsk. 32, No. 16 (1961).

9. Jensen A.S., Hansen P.G., and Jonson B., Nucl. Phys. A431, 393 (1984).

10. Dobaczewski J., and Dudek J., Comp. Phys. Commun. 102, 166, 183 (1997), and to be published.

11. Beiner M., Flocard H., Nguyen Van Giai, and Quentin P., Nucl. Phys. A238, 29 (1975).

12. Strutinsky V.M., Nucl. Phys. A218, 169 (1974).

13. Bohr A. and Mottelson B.R., Nuclear Structure, vol. 1, New York: W.A. Benjamin, 1969.

14. Clemenger K., Phys. Rev. B32, 1359 (1985).

15. Manninen M., Mansikka-aho J., Nishioka H., and Takahashi Y., Z. Phys. D31, 259 (1994).

16. Yannouleas C., and Landman U., Phys. Rev. B51, 1902 (1995). 
17. Shlomo S., Nucl. Phys. A539, 17 (1992).

18. Dobaczewski J., Nazarewicz W., and Satuła W., to be published.

19. Myers W., and Swiatecki W., Nucl. Phys. A81, 1 (1966).

20. Abboussir Y., Pearson J.M., Dutta A.K., and Tondeur F., Atomic Data and Nuclear Data Tables 61, 127 (1995)

21. Myers W., and Swiatecki W., Nucl. Phys. A612, 249 (1997).

22. Satuła W., Dean D.J., Gary J., Mizutori S., and Nazarewicz W., Phys. Lett. B407, 103 (1997).

23. Satuła W., Dean D.J., Dobaczewski J., Garrett J., and Nazarewicz W., to be published.

24. Myers W., Droplet Model of Atomic Nuclei, New York: Plenum, 1977.

25. Brenner D.S, Wesselborg C., Casten R.F., Warner D.D., and Zhang J.-Y., Phys. Lett. B243, 1 (1990).

26. Satuła W., and Wyss R.A., Phys. Lett. B393, 1 (1997).

27. Rosenfeld L., Nuclear Forces, Amsterdam: North-Holland, 1948.

28. N. Anantaraman N., and Schiffer J.P., Phys. Lett. B37, 229 (1971), and Schiffer J.P., Ann. Phys. 66, 78 (1971).

29. Satuła W., and Wyss R., to be published.

30. Tajima N., Takahara S., and Onishi N., Nucl. Phys. A603, 23 (1996).

31. Goodman A.L, these proceedings.

32. Engel J., Langanke K., and Vogel P., Phys. Lett. B389, 211 (1996).

33. Dobaczewski J., and Dudek J., Phys. Rev. C52, 1827 (1995).

34. Engel Y.M., Brink D.M., Goeke K., Krieger S.J., and D. Vautherin, Nucl. Phys. A249, 215 (1975).

35. Zhang J.-Y., Casten R.F., and Brenner D.S., Phys. Lett. B227, 1 (1989).

36. Satuła W., Dobaczewski J., and Nazarewicz W., to be published. 